$3^{\mathrm{e}}$ numéro_Les Annales de QPES

\title{
Accompagner les étudiants bacheliers bioingénieurs dans le développement de leurs compétences transversales
}

\author{
Charlotte Descamps \\ UCLouvain, Earth and Life Institute, Louvain-la-Neuve, Belgique \\ (charlotte.descamps@uclouvain.be) \\ FRANÇOIS GASPARD \\ UCLouvain, Earth and Life Institute, Louvain-la-Neuve, Belgique \\ (francois.gaspard@uclouvain.be) \\ PIERRE DELMELLE \\ UCLouvain, Earth and Life Institute, Louvain-la-Neuve, Belgique \\ (pierre.delmelle@uclouvain.be) \\ ANNE-LAURE JACQUEMART \\ UCLouvain, Earth and Life Institute, Louvain-la-Neuve, Belgique \\ (anne-laure.jacquemart@uclouvain.be) \\ JULIE LECOQ \\ UCLouvain, Louvain Learning Lab, Louvain-la-Neuve, Belgique \\ (julie.lecoq@uclouvain.be)
}

\section{Résumé}

Cet article propose de décrire la conception, le déroulé et l'évaluation de quatre modules de formation aux compétences transversales instaurés dans le cadre d'une réforme du programme de bachelier dans la Faculté des bioingénieurs de l'UCLouvain en réponse à deux enjeux facultaires : 1) intégrer les disciplines dans un contexte plus appliqué et 2) développer les compétences transversales des futurs bioingénieurs. Le scénario des modules prenait pour point d'ancrage la connaissance de soi et la conscience des différences interpersonnelles et visait un développement progressif des compétences de collaboration, d'organisation et de communication, en articulation directe à la réalisation d'un projet. Quarante-quatre équipes d'étudiants ont pris part au dispositif. Celui-ci a fait l'objet d'une évaluation quantitative (enquête) et qualitative (focus group). Les résultats intègrent des mesures de l'efficacité du groupe et des émotions vécues.

\footnotetext{
Abstract

This paper proposes to describe the design, development and evaluation of four soft skills's training modules introduced as part of a curriculum reform in the Faculty of Bioengineers in
} 
UCLouvain in response to two issues: integrate the disciplines in a more applied context and develop the softs skills of future bioengineers. The scenario of the modules was based on selfknowledge and awareness of interpersonal differences and aimed at a progressive development of collaboration, organization and communication skills, directly linked to the realization of a project. Forty-four groups of students took part in the program. It was subject to a quantitative (survey) and qualitative (focus group) evaluation. The results include measures of group effectiveness and emotions experienced.

\section{Mots-clés}

Apprentissage collaboratif, projet,compétences transversales, sciences environnementales

\section{Keywords}

Collaborative learning, project, soft skills, environmental sciences

\section{Introduction}

Le dispositif mis en place est le résultat d'un processus de co-construction entre plusieurs acteurs. Les assistants du cours, principaux protagonistes, ont demandé à être accompagnés par une conseillère pédagogique du service d'appui à l'enseignement de l'UCLouvain (Université catholique de Louvain), le Louvain Learning Lab. Au même moment, celle-ci animait une recherche collaborative sur l'accompagnement des travaux de groupes avec des enseignants issus de diverses facultés. L'objectif de la recherche était d'expérimenter divers outils de gestion de la dynamique d'équipe documentés dans une communauté de pratiques. Ces outils (Crahay et Lecoq, 2017) ont été proposés aux assistants qui s'en sont emparés pour élaborer un dispositif pédagogique d'accompagnement d'équipes d'étudiants bioingénieurs dans la réalisation d'un projet. Cet article propose un retour critique et documenté sur l'expérience mise en place par les assistants. Après avoir décrit le contexte justifiant la création de ce dispositif, ses points d'ancrages théoriques seront présentés avant d'en décrire plus précisément le contenu et enfin l'impact. Ce dernier fera l'objet d'une discussion et d'une mise en perspective.

\section{Contexte et problématique}

En 2017, dans le cadre d'une réforme du programme de bachelier de la Faculté des bioingénieurs de l'UCLouvain, un nouveau cours a été créé afin de répondre à deux enjeux facultaires : 1) intégrer les disciplines dans un contexte plus appliqué et 2) développer les 
compétences transversales des futurs bioingénieurs. Plus précisément, il s'agissait de réviser entièrement le cours de systématique végétale de manière à imaginer un scénario pédagogique susceptible de relever ces deux défis en répondant à une formule de 30 heures de cours théorique et 30 heures de travaux pratiques (5 ECTS).

Les enseignants et leurs assistants ont décidé de concevoir le dispositif pédagogique autour d'un projet d'équipe avec pour objectif la réalisation d'un diagnostic environnemental à partir d'une excursion sur terrain réel. Concrètement, durant un quadrimestre, les étudiants choisissaient eux-mêmes un site naturel (parcelle forestière, prairie, verger...) et devaient déterminer la nature du sol, réaliser et analyser des prélèvements, identifier les plantes rencontrées et constituer un herbier des plantes les plus représentatives. Le choix d'un tel projet se justifiait en ce qu'il permettait d'appliquer directement des notions de systématique végétale et de pédologie en intégrant des concepts liés à l'écologie, à la biogéographie et à la gestion des milieux. L'objectif du cours était de mettre en relation le type de sol avec la végétation et de poser un avis critique quant à la gestion du site.

Pour ces étudiants de 2e bachelier en bioingénieur, il s'agissait non seulement du premier travail de terrain, mais aussi de leur première expérience de travail en équipe. En effet, les différentes étapes du projet se réalisaient par équipe de quatre ou cinq étudiants. Si le travail de terrain était soutenu par des séances de travaux pratiques en systématique végétale, il a été décidé d'offrir un accompagnement au travail d'équipe afin de faciliter le développement des compétences transversales visées par le cours : la gestion de projet (planification et respect des échéances), la communication scientifique (orale et écrite), la capacité à construire une dynamique relationnelle favorable, la connaissance de soi et d'autrui et la réflexivité. En collaboration avec une conseillère pédagogique, quatre modules d'accompagnement ont été élaborés pour répondre à ces enjeux : 1) «apprendre à se connaître pour mieux travailler en équipe », 2) «apprendre à s'organiser » et 3) et 4) «apprendre à communiquer» (deux modules). Ces modules se clôturaient par une séance de bilan réflexif quant aux compétences développées.

\section{Perspective théorique}

Dans la réalisation d'un projet, composer des équipes d'étudiants rencontre de nombreux avantages (Kapp, 2009) : une productivité supérieure au travail individuel, davantage de 
motivation et de persistance en cas d'obstacle, le développement d'aptitudes collaboratives, communicationnelles et de résolution de problème très utiles et recherchées aujourd'hui.

Si les atouts de la collaboration sont bien connus, les difficultés du travail en équipe le sont tout autant. Verzat, O'Shea et Raucent (2015) ont relevé plusieurs obstacles à la collaboration : un manque de confiance entre les membres (Bianey, Ruiz et Adams, 2004 ; Huff, Cooper et Jones, 2002), la tendance de certains à s'appuyer sur les autres sans contribuer à la tâche (Oakley, Hanna, Kuzmyn et Felder, 2007), la différence de niveaux d'engagement et de compétences au départ (Duek, 2000), ou encore la discrimination raciale ou sexuelle (Cox, 1996 ; Faidley, Salisbury-Glennon, Glenn et Hmelo, 2000). Il ne suffit pas de mettre des personnes ensemble pour les voir collaborer de manière efficace et féconde. Il est nécessaire que le travail en équipe soit structuré et accompagné pour qu'il puisse atteindre son potentiel pédagogique (voir Raucent et Vander Borgh, 2006).

C'est dans cet objectif que, dans le cadre de ce cours, il a été décidé de concevoir des modules visant à accompagner les étudiants dans l'acquisition de compétences à la fois organisationnelles, communicationnelles et de gestion de projet, mais également dans le développement de compétences relationnelles et réflexives axées sur la connaissance de soi et des autres. Nous parlerons ici d'équipe plutôt que de groupe puisque les étudiants ont été mis ensemble en vue de l'atteinte d'un objectif clair qu'ils ont contribué à définir ensemble.

\subsection{Comment accompagner les équipes ?}

Traditionnellement, l'accompagnement des équipes s'est fait par la distribution de rôles au sein d'une équipe ou par la prise de conscience que les membres de l'équipe jouent un rôle. Moreno (1965) définit le rôle comme la «manière d'être et d'agir que l'individu assume au moment où il réagit à une situation donnée dans laquelle d'autres objets ou personnes sont engagés ». On distingue souvent deux types de rôles : les rôles centrés sur la tâche qui visent à organiser et à coordonner l'effort de l'équipe (par exemple : l'animateur, le secrétaire, le gardien du temps, etc.) et les rôles centrés sur «l'entretien de l'équipe » qui visent à créer un climat relationnel favorable (par exemple : le médiateur, le protecteur, le visionnaire, etc.). Aujourd'hui, des recherches montrent un intérêt croissant pour les variables intrapersonnelles (styles cognitifs ou traits de personnalité) dont l'importance se révèle au-delà des rôles : dans la manière personnelle d'exprimer ce rôle, dans les spécificités à travers lesquelles chacun va endosser sa fonction au sein de l'équipe (Oberlé et Drozda-Senkowska, 2006). 
La conception des modules dont il est question ici s'est inscrite dans cette perspective en mettant les étudiants en situation d'expérimenter leurs différences et en leur donnant la possibilité de recevoir un éclairage sur celles-ci par le recours à un modèle de personnalité (ComColor®; https://www.comcolors.com/be/).

\subsection{Le pari de la connaissance de soi et des autres}

Longtemps, les recherches se sont focalisées sur les éléments fonctionnels susceptibles de rendre la tâche plus efficace plutôt que sur les éléments relationnels et cela pour diverses raisons (Oberlé et Drozda-Senkowska, 2006). Tout d'abord, les dimensions relationnelles sont des variables méthodologiquement beaucoup moins simples à délimiter et à manipuler. Ensuite, l'autre, différent de soi, a été longtemps considéré pour la diversité de ses idées, la controverse, le débat et l'avènement d'idées nouvelles; beaucoup plus rarement dans sa dimension personnelle ou affective, suspectée de «polluer » les débats.

Ces dernières années, l'évolution importante du courant du développement personnel a progressivement proposé de mettre en lumière ces différences interpersonnelles de manière à les reconnaître, les respecter et à désamorcer leurs effets implicites (Hendry et al., 2005). Il a été décidé d'initier les étudiants à un modèle de personnalité relativement simple (ComColor ${ }^{\circledR}$; https://www.comcolors.com/be/) afin de leur offrir une grille de lecture visant à activer la prise de conscience de leur fonctionnement personnel, tout en les ouvrant à une compréhension respectueuse de la différence des autres. Vécue comme une opportunité et non comme une menace, la différence interpersonnelle pouvait alors devenir une richesse et impacter favorablement le climat émotionnel et la performance de l'équipe (Miller, Trimbur et Wilkes, 1994).

\section{Description du dispositif}

En tout, 178 étudiants étaient inscrits au cours de «Projet intégré en diagnostic environnemental ». Ce cours se déclinait en plusieurs parties : 22 heures de cours magistral, une excursion, quatre séances de travaux pratiques de systématique végétale, quatre modules de «dynamique de groupe » et une séance de présentation orale de leur projet (sous format «Mon projet en 180 secondes »). Il se déroulait sur un quadrimestre (Tableau 1). 
Tableau 1 : Déroulé du cours « Projet intégré en diagnostic environnemental »

\begin{tabular}{|lc|}
\hline \multicolumn{1}{|c}{ Activités } & Heures \\
\hline Cours magistral & 22 \\
Excursion & 2 \\
Travaux pratiques de systématique végétale & 8 \\
Dynamique de groupe : Module 1: Travailler en équipe & 2 \\
Dynamique de groupe : Module 2: Apprendre à s'organiser en équipe & 2 \\
Dynamique de groupe : Module 3: Apprendre à communiquer (au sein de l'équipe) & 2 \\
Dynamique de groupe : Module 4: Apprendre à communiquer (vers l'extérieur) & 2 \\
Présentation finale orale "Mon projet en 180 secondes" & 2 \\
\hline
\end{tabular}

Les 178 étudiants étaient répartis en six groupes horaires, eux-mêmes composés de six équipes de quatre ou cinq étudiants (soit 44 équipes d'étudiants au total). Selon une approche similaire à celle proposée par Edward Kapp (2009), le scénario des quatre modules de «dynamique de groupe » a été construit de manière à respecter les trois étapes suivantes :

- Familiariser les étudiants avec leurs préférences personnelles au travail et les conscientiser au respect des différences interpersonnelles ;

- Amorcer le travail en équipe par l'élaboration d'un contrat d'équipe permettant de partager les forces de l'équipe et de s'engager quant aux résultats visés et aux modalités de fonctionnement convenues ensemble ;

- Utiliser l'évaluation par les pairs de manière à réguler le travail en cours.

Conformément aux objectifs du cours, l'évaluation certificative visait à attester l'intégration de compétences «appliquées » par l'appréciation du projet d'analyse du site et par la confection d'un herbier. Ainsi que présenté dans le Tableau 2, l'évaluation finale du cours valorisait les productions relatives aux modules de dynamique de groupe à concurrence de $45 \%$ de la note globale. Ces productions étaient notées sur base de leur réalisation (remis ou pas) et du soin porté à celle-ci (travail bâclé ou pas). La description et les objectifs des travaux liés aux modules de dynamiques de groupes sont détaillés dans les sections suivantes. 
Tableau 2 : La note finale du cours sur 20 points

Présentation orale
«Mon site en 180 secondes »

Fiche finale + Herbier

$25 \% 5 \mathrm{pts}$

$25 \% 5 \mathrm{pts}$

\begin{tabular}{|c|c|c|c|c|c|c|}
\hline \multirow[b]{2}{*}{$\begin{array}{c}\text { TP de } \\
\text { systématique } \\
5 \% 1 \mathrm{pt}\end{array}$} & \multicolumn{6}{|c|}{ Modules « dynamique de groupe » } \\
\hline & $\begin{array}{c}\text { Contrat } \\
\text { d'équipe } \\
5 \% \\
1 \mathrm{pt}\end{array}$ & $\begin{array}{c}\text { Choix } \\
\text { du site } \\
5 \% \\
1 \mathrm{pt}\end{array}$ & $\begin{array}{c}\text { Retour } \\
\text { Réflexif } \\
10 \% \\
2 \text { pts }\end{array}$ & $\begin{array}{c}\text { Gantt } \\
\text { Chart } \\
5 \% \quad 1 \mathrm{pt}\end{array}$ & $\begin{array}{c}\text { Présentation } \\
\text { brouillon } \\
5 \% 1 \mathrm{pt}\end{array}$ & $\begin{array}{c}\text { Présentation } \\
\text { intermédiaire } \\
15 \% 3 \text { pts }\end{array}$ \\
\hline
\end{tabular}

Pour sensibiliser les étudiants à l'intérêt de ces modules dans leur parcours de formation, un clip vidéo d'interviews de bioingénieurs travaillant dans des domaines divers et témoignant chacun du management d'équipe comme d'une compétence essentielle et indispensable de leur métier leur a été présenté.

De plus, le scénario pédagogique des modules de dynamique de groupe a été conçu en étroite articulation avec la réalisation progressive du projet disciplinaire. Chacun des quatre modules durait deux heures et visait un objectif spécifique tel que décrit dans les sections suivantes.

\subsection{Module 1 : «Travailler en équipe »}

L'objectif de ce premier module de deux heures était d'entreprendre le travail des équipes en leur faisant expérimenter les étapes permettant de faire évoluer une collection d'individus en une équipe d'individus interdépendants. À cette fin, plusieurs activités ont été proposées aux étudiants :

- La séance a débuté par un jeu brise-glace visant à leur permettre de faire connaissance entre eux et de faire émerger la variété des profils et des préférences de travail ;

- À partir de la mise en évidence de cette diversité, les assistants ont composé les équipes en leur donnant comme tâche de se remémorer individuellement une expérience de bonne collaboration dans leur vie personnelle et d'en dégager les dimensions principales. Ces expériences ont alors été partagées au sein des équipes ;

- Un modèle de personnalité à six couleurs visant à stimuler la connaissance de soi et des autres, le modèle ComColor® a alors été présenté aux étudiants. Il a ensuite été proposé aux étudiants qui étaient intéressés, de passer le questionnaire de ComColor®, afin de déterminer la/les couleur(s) principale(s) correspondant à leur type de personnalité ; 
- Enfin, une typologie des fonctions (rôles) dans une équipe a été présentée aux étudiants en vue de les outiller dans la planification de leurs réunions de travail.

La séance s'est terminée par une pose pour la réalisation des photos de chaque équipe.

À l'issue du module, deux premières productions ont été demandées : la rédaction d'un contrat d'équipe sous la forme d'une charte signée par chacun des membres et reprenant les points forts, points faibles de chacun, les résultats visés collectivement et les modalités de fonctionnement. Les équipes ont également reçu la tâche de choisir librement un site d'étude en Belgique et de se l'approprier en se répartissant les tâches : prise de contact avec le propriétaire de la parcelle, collecte de données, recherches bibliographiques.

\subsection{Module 2 « Apprendre à s'organiser en équipe »}

Afin d'outiller les étudiants dans l'organisation et la planification des étapes de leur projet, les équipes ont participé à un second module d'une durée de deux heures avec pour objectif de sensibiliser les étudiants sur trois aspects : l'organisation et le déroulé des réunions, la gestion de l'information et la planification de projet.

- Lors d'une première activité, les équipes ont dû dégager les ingrédients d'une réunion efficace. Ces éléments ont ensuite été restructurés sur une échelle temporelle (avant, pendant et après la réunion) au cours d'un brainstorming collectif ;

- Pour sensibiliser les étudiants à l'importance des compétences organisationnelles pour leur future profession, les assistants leur ont montré des capsules vidéo d'interviews de bioingénieurs diplômés et expérimentés afin de souligner l'importance d'une bonne organisation et planification en gestion de projet ;

- Un outil a ensuite été proposé aux étudiants (le diagramme de Gantt) afin de les outiller concrètement dans la gestion des informations et la planification des étapes du travail. Une expérience personnelle d'usage a été évoquée par l'un des assistants et d'autres témoignages de diplômés ont été présentés puis synthétisés avant de laisser le temps aux équipes de travailler sur l'organisation et la planification de leur propre projet ;

- À la fin du module, les équipes ont reçu une tâche à réaliser pour la prochaine séance : Individuellement, à l'aide d'un outil d'auto-positionnement (Figure 1), il a été demandé à chaque étudiant de réaliser une évaluation du fonctionnement de son équipe en positionnant l'équipe sur les sept dimensions suivantes : production de l'équipe, climat de travail, communication de chacun, répartition des rôles, apprentissages réalisés, participation et gestion des conflits. Chaque étudiant a également dû rédiger un bref 
bilan réflexif quant à sa satisfaction ou son insatisfaction par rapport à l'avancement du projet et la collaboration dans l'équipe.

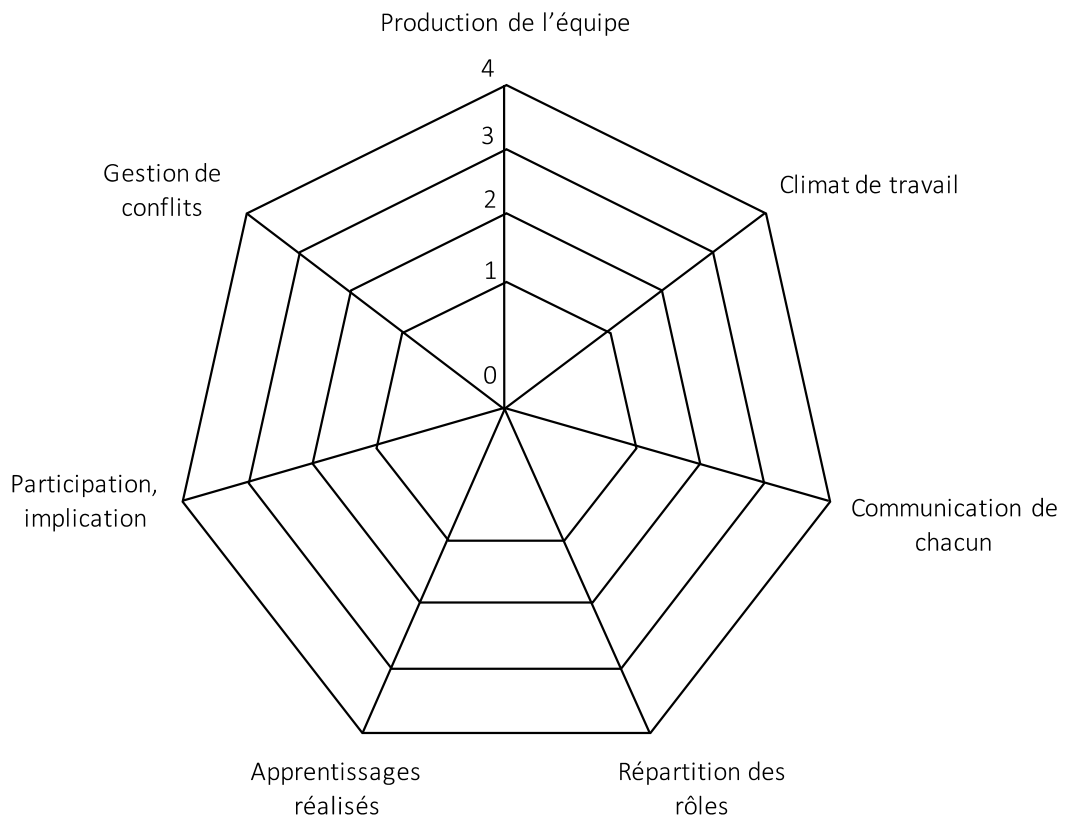

Figure 1 : Outil d'auto-positionnement (inspiré de Crahay et Lecoq, 2017, p. 43)

\subsection{Module 3 « Apprendre à communiquer (au sein de l'équipe)»}

Les étudiants ont ensuite participé à un troisième module dont l'objectif était double. Tout d'abord, il visait, à mi-parcours, à désamorcer les difficultés relationnelles éventuelles en proposant un exercice de communication sur le fonctionnement de leur équipe. Ensuite, il s'agissait d'outiller les étudiants en matière de communication de leur projet vers l'extérieur.

Il a d'abord été demandé aux équipes de confronter leurs outils d'auto-positionnement de manière à en observer les divergences. Chaque discordance rencontrée a donné lieu à un point de discussion en vue de pouvoir répondre aux questions suivantes : "Quels sont les éléments dont nous sommes fiers? Quels sont ceux que l'on souhaiterait améliorer au niveau de notre fonctionnement en équipe et sur lesquels nous nous engageons à travailler pour la suite du projet ? ». Enfin, les étudiants ont dû réfléchir aux conditions rendant possible un tel exercice. 
Sur base des éléments émergeant de l'exercice, les assistants ont réalisé une restructuration autour de la notion de feedback en introduisant quelques grands principes issus de la communication non violente proposés par Rosenberg (1999).

Le module s'est conclu par une discussion collective visant à dégager les «trucs et astuces » d'une présentation efficace en vue de la présentation «intermédiaire » de leur projet. Un brouillon de cette présentation leur a été demandé en vue du Module 4.

\subsection{Module 4 « Apprendre à communiquer (vers l'extérieur)»}

Cette dernière séance de deux heures a permis à chaque équipe de s'essayer à présenter les premiers éléments de leur projet. Outre le fait qu'il s'agissait d'une balise intéressante pour engager les équipes à avancer dans leur travail, cette séance poursuivait plusieurs objectifs :

- Placer les étudiants dans une situation de communication proche de celle qu'ils auraient à l'examen, mais aussi plus tard, dans leur vie professionnelle ;.

- Donner l'opportunité de recevoir des feedbacks de leurs pairs et de leurs assistants ;

- S'essayer à la formulation de questions et de feedbacks pertinents ;

- Dégager, grâce à la vision de multiples présentations et à la réception de multiples feedbacks, les éléments d'une présentation de qualité.

Pour la séance, chaque équipe a été jumelée à deux autres équipes : une premier chargée de poser des questions de clarification sur le projet, une seconde chargée de formuler une évaluation sous forme d'un feedback qualitatif (fond et forme). Une grille d'évaluation critèriée a été fournie à tous de manière à faciliter l'exercice. Les présentations se sont succédé, suivies chaque fois des interventions des équipes questionneurs et évaluateurs. En l'absence volontaire de consignes précises, chaque présentation a été porteuse de diversité et d'originalité. La séance s'est clôturée par une mise en évidence collective des éléments facilitateurs de la communication du projet.

Les assistants ont rédigé une évaluation précise pour chacune des équipes. Cette fiche d'évaluation personnalisée leur a ensuite été remise pour les aider à réaliser la présentation finale « Mon site en 180 secondes $»^{1}$.

\footnotetext{
${ }^{1}$ Les consignes pour présenter le site en 180 secondes étaient les suivantes : 3 diapos, 3 minutes, une présentation attractive et synthétique. Afin de maintenir l'audience attentive, un vote a été réalisé à l'issue de la séance pour élire la meilleure présentation.
} 


\subsection{Bilan réflexif}

Au terme du parcours, après la présentation finale du projet devant les professeurs, les équipes se sont retrouvées une dernière fois pour une séance collective d'évaluation du dispositif de deux heures. L'objectif de la séance était de faire le point sur le dispositif pédagogique en (1) leur proposant une activité où elles devaient tracer leur ressenti sur une ligne du temps du quadrimestre (avec le 0 en ordonnée correspondant à un état « neutre », la surface au-dessus de l'ordonnée correspondant à un état «motivé » et la surface en dessous de l'ordonnée correspondant à un état « démotivé » et (2) en engageant un débat afin de faire remonter les points positifs et négatifs des modules de dynamiques de groupe.

\section{Analyse}

\subsection{Objectifs de l'analyse}

L'équipe enseignante et les assistants ont souhaité recueillir le feedback des étudiants quant à leur appréciation du dispositif, sa cohérence au sein du cours (1) et quant à la manière dont les équipes avaient fonctionné, tant sur le plan de l'efficacité dans la gestion du projet (2) que sur le vécu émotionnel (3). Mais leur objectif principal a été de vérifier l'adéquation du dispositif d'accompagnement des équipes avec le développement des compétences transversales visées par le cours (4). Au nombre de sept, celles-ci ont été formulées comme suit :

- Mener un projet en équipe, en planifiant les étapes et la coordination de ce projet en fonction d'objectifs prédéfinis et d'échéances qui sont imposées ;

- Communiquer par l'intermédiaire d'un rapport écrit les résultats et conclusions principaux relatifs à la réalisation d'un projet, en suivant un canevas rigoureux et en utilisant un style et une forme respectant les standards scientifiques ;

- Communiquer oralement le résultat d'un projet devant une audience ;

- Construire une dynamique relationnelle favorable dans une équipe de travail ;

- Mettre en place les conditions propices à une bonne dynamique d'équipe ultérieurement, dans d'autres travaux de groupe ;

- Me connaître et me donner les moyens d'évoluer personnellement ;

- Connaître les autres et ajuster mes interventions en tenant compte d'eux.

\subsection{Mesures}

Afin d'enrichir le recueil d'informations, celui-ci s'est déroulé selon une approche mixte (quantitative et qualitative). Un questionnaire (voir l'Annexe) a été proposé en ligne 
avant la séance de bilan réflexif. En tout, 141 étudiants sur 178 y ont répondu, soit une participation de 79,2\%. Ce questionnaire était composé de questions fermées à choix multiples et de questions ouvertes. Lors de la séance de bilan réflexif, deux focus groups de respectivement 15 et 17 ont été réalisés afin de recueillir des données plus exploratoires, issues de la discussion entre étudiants appartenant à des sous-équipes différentes et se proposant sur base volontaire. Il s'agissait de discuter de la pertinence, du séquençage des diverses activités et productions proposées dans le cadre des 4 modules. Ces focus groups ont duré chacun une cinquantaine de minutes.

\subsubsection{Satisfaction des étudiants et cohérence des quatre modules au sein du cours}

Sur une échelle de Likert allant de 1 «pas du tout d'accord » à 4 « tout à fait d'accord », les étudiants ont dû se prononcer quant à leur appréciation des modules d'accompagnement du projet et quant à la cohérence de leur articulation avec le cours. En vue de recueillir davantage d'éléments, un espace de commentaires ouverts leur suggérait de préciser leur réponse. Concernant les quatre modules, trois autres espaces de commentaires ont permis aux étudiants d'en mentionner les points positifs, les pistes d'amélioration et des remarques à communiquer aux assistants. Les données recueillies au sein des deux focus groups ont permis un recueil encore plus approfondi.

\subsubsection{Efficacité groupale}

Ces dimensions ont été tirées d'une traduction française de l'échelle de mesure d'appréciation de l'efficacité d'une équipe (Ohland et al., 2012), mieux connue sous les initiales CATME (Comprehensive Assessment of Team Member Effectiveness). Selon les auteurs, l'efficacité groupale se définit par cinq dimensions: la satisfaction groupale, l'interdépendance, l'apprentissage, la productivité et la qualité du climat de l'équipe. Chacune de ces dimensions a été mesurée par trois items à l'exception de la dernière catégorie (Tableau 3). Les étudiants devaient se prononcer sur une échelle de Likert à quatre points allant de «pas du tout d'accord » à « tout à fait d'accord » sur chacun des items du Tableau 3. 
Tableau 3 : Mesure de l'efficacité groupale

\begin{tabular}{|l|l|}
\hline $\begin{array}{l}\text { Degré de } \\
\text { satisfaction avec } \\
\text { l'équipe }\end{array}$ & $\begin{array}{l}\text { JE SUIS SATISFAIT DES MEMBRES DE MON GROUPE } \\
\text { JE SUIS SATISFAIT DE LA MANIĖRE DONT ON A TRAVAILLÉ ENSEMBLE } \\
\text { JE SUIS CONTENT DANS CE GROUPE }\end{array}$ \\
\hline $\begin{array}{l}\text { Degré } \\
\text { d'interdépendance } \\
\text { de l'équipe }\end{array}$ & $\begin{array}{l}\text { AFIN DE COMPLÉTER NOS TRAVAUX, MES COÉQUIPIERS ET MOI DEVIONS } \\
\text { COMPTER LES UNS SUR LES AUTRES POUR DES INFORMATIONS ET DES } \\
\text { C'AVAIS BESOIN DE MES COÉQUIPIERS POUR RÉALISER LE TRAVAIL } \\
\text { J'AI EU L'IMPRESSION DE TRAVAILLER EN SOLO (R) }\end{array}$ \\
\hline $\begin{array}{l}\text { L'apprentissage } \\
\text { dans l'équipe }\end{array}$ & $\begin{array}{l}\text { MES COÉQUIPIERS ET MOI AVONS ÉCHANGÉ ET PARTAGÉ DES INFORMATIONS, } \\
\text { MES COÉQUTIPIERS ET MOI AVONS TESTÉ LES IDÉES DU GROUPE } \\
\text { MES COÉQUIPIERS ET MOI AVONS RÉFLÉCHI ENSEMBLE POUR RESTRUCTURER } \\
\text { NOS CONNAISSANCES ET AFFINER NOTRE COMPRÉHENSION }\end{array}$ \\
\hline $\begin{array}{l}\text { La qualité de la } \\
\text { production de } \\
\text { l'équipe }\end{array}$ & $\begin{array}{l}\text { MES COÉQUIPIERS ET MOI ÉTIONS CONSTAMMENT FOCALISÉS SUR L'OBJECTIF } \\
\text { AT ATEINDRE } \\
\text { MES COÉQUIPIERS ET MOI AVONS TOUJOURS RESPECTÉ LES DÉLAIS IMPOSÉS } \\
\text { MES COÉQUIPIERS ET MOI AVONS PRODUIT UN TRAVAIL DE QUALITÉ }\end{array}$ \\
\hline $\begin{array}{l}\text { Climat de } \\
\text { l'équipe }\end{array}$ & $\begin{array}{l}\text { DANS NOTRE GROUPE, NOUS AVONS RÉUSSI À ÉTABLIR UN CLIMAT DE } \\
\text { CONFIANCE } \\
\text { DANS NOTRE GROUPE, NOUS AVONS GÉRÉ DE FAÇON CONSTRUCTIVE LES } \\
\text { DIFFICULTÉS QUI SONT SURVENUES }\end{array}$ \\
\hline
\end{tabular}

\subsubsection{Vécu émotionnel}

La qualité du vécu personnel au sein des équipes a été principalement explorée lors des focus groups. Néanmoins, une mesure des émotions vécues a été choisie en tant qu'indicateur du climat relationnel. À cette fin, une traduction française du questionnaire Differential Emotionnal Scale ${ }^{2}$ de Izard et ses collègues (Izard et al., 1974) a été utilisée et adaptée ${ }^{3}$ en proposant aux étudiants de cocher les 5 états émotionnels résumant le mieux comment ils se sont sentis au sein de leur équipe.

\subsubsection{Développement des compétences transversales}

Les étudiants devaient estimer leur degré de progression dans chacune des sept compétences transversales ciblées par le cours en positionnant pour chacune leur niveau de maîtrise «avant le cours» et «après le cours » sur deux échelles allant de zéro à dix $(0=$ «pas du tout compétent » et $10=$ «tout à fait compétent »). L'échelle à dix niveaux a été choisie de manière

\footnotetext{
${ }^{2}$ Cette traduction est issue de Schaefer et al, 2010.

${ }^{3}$ On a réduit le nombre d'émotions de 16 à 12 en enlevant les émotions moins susceptibles d'être rencontrées dans un tel contexte (par exemple : attendri ou dédaigneux) et en remplaçant amoureux par passionné, pleinement engagé).
} 
à donner un spectre de positionnement plus vaste aux étudiants et à augmenter les possibilités de contraste.

\section{Résultats}

Cette section reprend les résultats du recueil de données au regard des quatre objectifs qui l'ont orienté :

\subsection{De l'enthousiasme pour le dispositif}

Le dispositif mis en place a été bien apprécié. Le degré de satisfaction moyen s'élève à 3,33 sur une échelle allant de 0 à 4 . La perception de la cohérence entre les diverses parties du dispositif a recueilli un score moyen de 3,04 sur 4. Les commentaires des étudiants précisent et détaillent ces résultats : "C'était des moments qui permettaient d'intégrer la théorie », "il y avait une super articulation entre les trois parties », "c'était plein de conseils pour nos études, mais aussi pour plus tard».

\section{Les principaux points positifs relevés sont :}

- l'intérêt pour le dispositif («le fait de pouvoir travailler par équipe et communiquer avec plusieurs personnes, apprendre à travailler avec de nouveaux outils, comme les modèles de personnalité. Le fait que justement ces travaux pratiques soient "dynamiques". Et on a enfin réalisé quelque chose de concret en tant qu'étudiant») ;

- l'ambiance lors des modules («L'ambiance conviviale et l'originalité des sujets abordés. On a abordé des sujets sur lesquels on ne se penchait jamais dans les autres cours. Or, savoir travailler en équipe est indispensable »);

- l'enthousiasme des assistants («Assistants supers, dynamiques et sympas! Ça fait plaisir d'avoir des travaux pratiques donnés comme ça») ;

- l'utilité des outils proposés : Certains outils ont été utilisés plus que d'autres mais les étudiants ont été satisfaits de les découvrir tous ( J'ai beaucoup aimé le bilan de fonctionnement en groupe. J'avais sur moi toute la charge, ça m'a aidé à le dire aux autres et ça a permis aux autres de le percevoir »).

\section{Les points d'attention relevés sont :}

- la taille des équipes qui, lorsqu'elle est en dessous de 4, ne permet pas un réel travail sur la dynamique («Mieux vaut faire des plus grands groupes pour vraiment illustrer les mécanismes de dynamique. »); 
- la grille de critères utilisée lors de la séance de communication, souvent jugée trop complexe à utiliser ( Il y avait trop de critères. Il y avait trop de gradations dans les notes à attribue. Nous manquions de temps pour évaluer sérieusement »).

\subsection{Des équipes efficaces}

Afin de valider la cohérence interne des indicateurs de fonctionnement groupal, la cohérence de chaque sous-échelle a été vérifiée en effectuant une analyse de consistance interne (alpha de Cronbach). Les alphas obtenus étant supérieurs à 0,70 pour chacune des sous-échelles, on a pu calculer cinq indicateurs. Comme l'illustre le Tableau 4, la moyenne de chacun des indicateurs de fonctionnement groupal est supérieure à 3,3 sur une échelle allant de 1 à 4 . Les équipes ont en moyenne très bien fonctionné. Les résultats présentés dans le Tableau 2 montrent que les équipes se sont senties efficaces et productives. Les membres de l'équipe semblent avoir expérimenté la richesse de l'interdépendance positive et la qualité d'apprentissage qu'elle permet.

Tableau 4 : Moyenne et écart-type des indicateurs de fonctionnement groupal

\begin{tabular}{|l|c|c|c|c|c|}
\hline & $\mathrm{N}$ & Minimum & Maximum & Moyenne & Écart-type \\
\hline $\begin{array}{l}\text { Degré de satisfaction } \\
\text { avec l'équipe }\end{array}$ & 139 & 1,00 & 4,00 & 3,36 & 0,69 \\
\hline $\begin{array}{l}\text { Degré d'interdépendance } \\
\text { de l'équipe }\end{array}$ & 138 & 2,00 & 4,00 & 3,32 & 0,55 \\
\hline $\begin{array}{l}\text { L'apprentissage dans } \\
\text { l'équipe }\end{array}$ & 140 & 1,50 & 4,00 & 3,41 & 0,62 \\
\hline $\begin{array}{l}\text { La qualité de la } \\
\text { production de l'équipe }\end{array}$ & 139 & 1,33 & 4,00 & 3,32 & 0,54 \\
\hline Climat de l'équipe & 140 & 1,33 & 4,00 & 3,42 & 0,68 \\
\hline
\end{tabular}

Le climat d'équipe est l'indicateur dont le score est le plus élevé (3,42 sur 4) ce qui est déjà un révélateur d'un niveau de confiance interpersonnel important. Le point suivant le confirme.

\subsection{Un vécu émotionnel positif au sein des équipes}

Les résultats révèlent que les étudiants ont éprouvé au cours de leur travail des émotions largement positives. L'émotion la plus ressentie est l'intérêt, suivie par la joie et, enfin, la 
passion. Les émotions négatives sont pratiquement absentes du paysage émotionnel à l'exception du stress, néanmoins éprouvée moins souvent que la surprise (Figure 2).

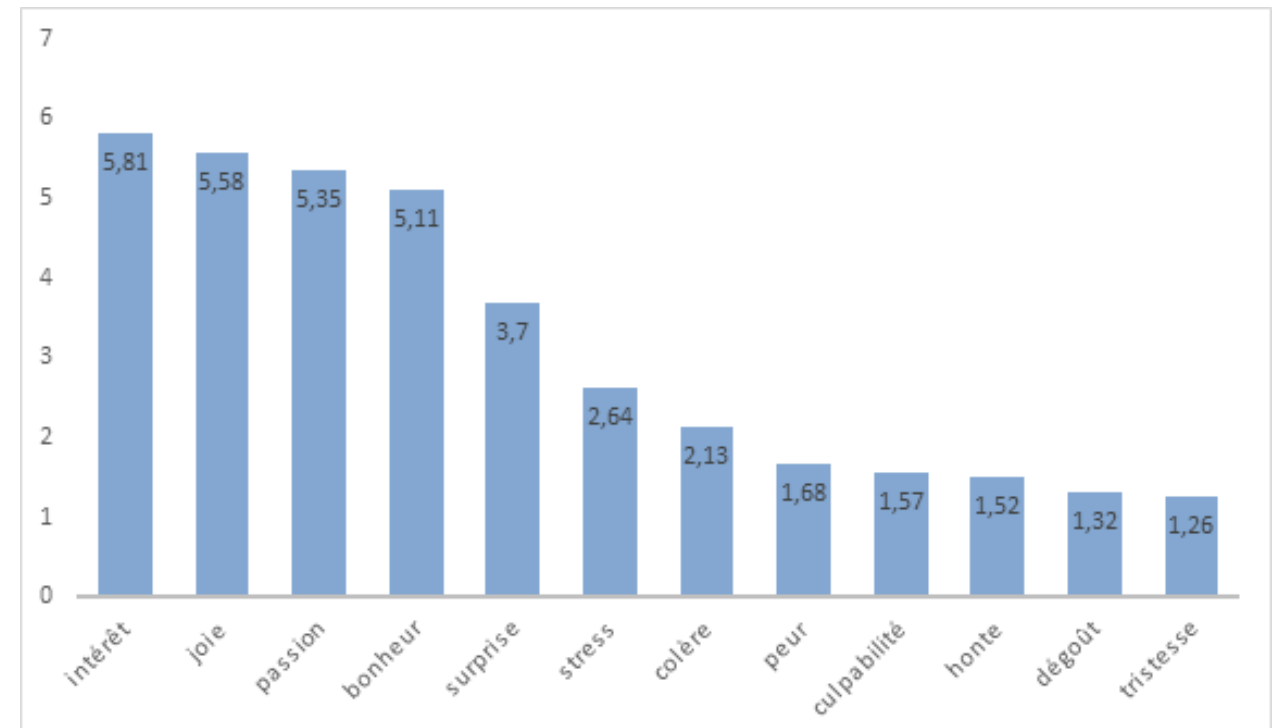

Figure 2 : Moyenne des émotions caractérisant le vécu des étudiants au sein de leur équipe

Les témoignages des étudiants présents lors des entretiens de focus groups ont confirmé ce vécu émotionnel positif :

- la joie : "La bonne ambiance », «la complicité entre les assistants», " une convivialité tout en restant vraiment sérieux »;

- l'intérêt/la surprise : «Un format comme ça, ça change! », "on n'est pas habitués à fonctionner ainsi, ça faisait du bien »;

- la passion/l'engagement : «On était dans du concret, enfin actifs », " c'était dynamique », «le côté participatif».

Au-delà des manifestations émotionnelles sont apparues d'autres dimensions intéressantes, révélatrices d'un encadrement dans lequel des étudiants se sont sentis en pleine confiance :

- la proximité : «Le tutoiement », «le fait que les assistants sont jeunes, proches de nous », « on n'a jamais eu un tel rapport avec des assistants »;

- la disponibilité : «Les assistants étaient toujours dispo, leur porte était ouverte, on pouvait leur poser toutes nos questions ». 


\subsection{Une progression subjectivement perçue sur chacune des compétences}

Les étudiants rapportent une évolution sur chacune des compétences transversales sondées (Tableau 5). Les compétences de communications sont celles qui montrent la plus belle progression $+1,98$ et $+2,28$ ). La capacité à gérer un projet s'est également bien consolidée $(+1,86)$.

La compétence pour laquelle la maîtrise s'avère la plus importante (9,10 en moyenne sur 10) au terme du cours est «Construire une dynamique relationnelle favorable dans une équipe de travail ». Les compétences de collaboration progressent ainsi de $+1,41$ et $+1,38$. Concernant notre pari sur la connaissance de soi, la progression perçue n'a pas été aussi importante qu'espéré avec $+0,86$ pour la connaissance de soi et $+1,27$ pour la connaissance des autres.

Deux explications peuvent être avancées :

- Ces compétences ont été jugées comme déjà bien maîtrisée dès le départ $(7,78$ et 7,74);

- L'utilisation du modèle de personnalité a peut-être souffert d'un usage trop superficiel. Pour des raisons éthiques, les assistants se sont limités à une initiation, proposant un approfondissement sur demande uniquement. Les focus groups ont révélé que si la majorité des étudiants avaient apprécié le modèle, certains l'ont jugé comme superflu, mettant en cause un usage trop restreint : "J'aurais voulu qu'on l'approfondisse plus », " on a tout de suite oublié nos couleurs », « on aurait dû mieux exploiter les résultats », «Vu si rapidement, ça fait trop quizz de magazine ».

\section{Tableau 5 : Développement des compétences transversales}

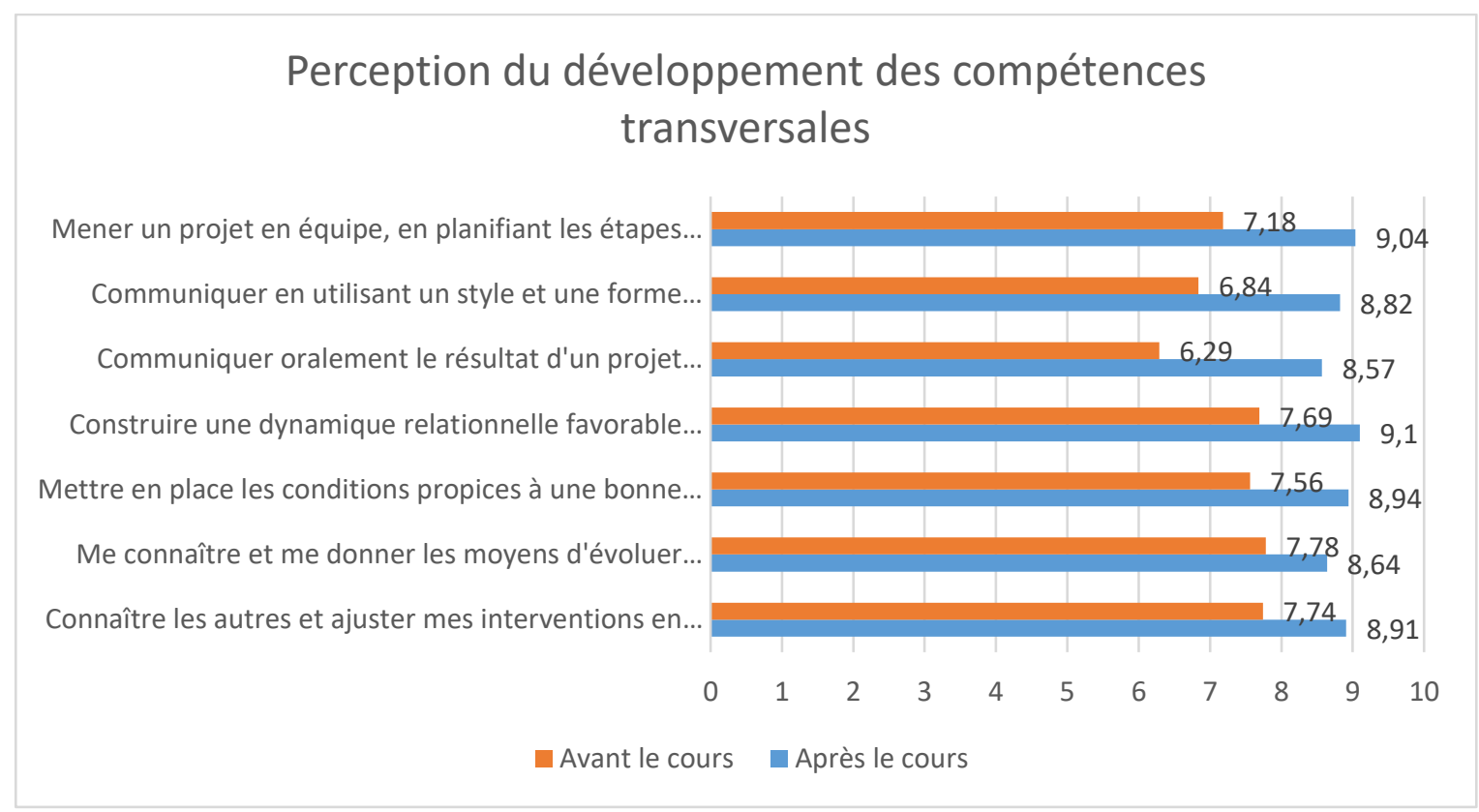




\section{Conclusion et Perspectives}

L'intérêt du dispositif présenté ici était de proposer un projet disciplinaire ancré sur le terrain et soutenu par un scénario d'accompagnement pédagogique conçu de manière à maximiser le développement des compétences transversales des étudiants bioingénieurs.

L'enquête et les focus groups révèlent l'enthousiasme des étudiants pour le dispositif et confirment les résultats du questionnaire qui rapporte un indicateur de performance groupal élevé, un climat émotionnel dominé par les émotions positives et la perception d'avoir progressé sur chacune des compétences visées.

Si ce dispositif pouvait donner lieu à une recherche, il serait intéressant de tester l'hypothèse selon laquelle une meilleure connaissance de soi et des autres favorise le climat émotionnel d'une équipe tout en contribuant ainsi à son efficacité. Si cette hypothèse se vérifiait, il conviendrait de s'interroger sur la part de variance expliquée par cette variable en comparaison à celle relative aux qualités du dispositif pédagogique lui-même (structure, cohérence, feedback, rôles des assistants, etc.).

Il serait tout aussi opportun d'explorer par une étude longitudinale la manière dont se construit la vie d'une équipe : quelles en sont les étapes principales ? Toutes les équipes passent-elles par les mêmes étapes ? Quelles sont les éventuelles configurations possibles ? Quels sont les points de difficulté ? Comment l'équipe s'organise-t-elle pour les surmonter ? Comment optimiser l'accompagnement dans ces moments clé ? Etc.

Sur le plan des émotions, seuls les vécus émotionnels individuels ont été sondés ici. Il serait pertinent d'envisager dans quelle mesure ces émotions peuvent s'observer au niveau groupal et par quel jeu d'influence émotions collectives et émotions singulières sont-elles susceptibles de s'harmoniser.

\section{Références bibliographiques}

Bianey, C., Ruiz, U. et Adams, S. G. (2004). Attitude toward teamwork and effective teaming. Team Performance Management, 10(7/8), 145-152

Cox, C. A. (1996). Student responses to problem-based learning in the Carribean. Communication au meeting de Research in Medical Education, Association of American Colleges, San Francisco, CA, avril. 
Crahay, M. et Lecoq, J. (2017). Accompagner des étudiant·e.s qui travaillent en groupe. Les cahiers du LLL, éditeur B. Raucent. Louvain-la-Neuve

Duek, J. E. (2000). Whose group is it anyway? Equity of student discourse in problem based-learning? Dans D. H. Evensen, and C. E. Hmelo (Dir.) : Problem-based learning. A research perspective on learning interactions. New Jersey : Lawrence Erlbaum Associates, Publishers.

Faidley, J., Salisbury-Glennon, J., Glenn, J. et Hmelo, C. E. (2000). How are we doing? Methods of assessing group processing in a problem-based learning context. Dans D. H. Evensen et C. E. Hmelo (dir.) Problem-based learning, a research perspective on learning interactions (p. 75-107). Hillsdale, NJ: Lawrence Erlbaum Associates.

Hendry, G. D., Heinrich, P., Lyon, P. M., Barratt, A. L., Simpson, J. M., Hyde, S. J., Gonsalkorale, S., Hyde, M., et Mgaieth, S. (2005). Helping students understand their learning styles: Effects on study self'efficacy, preference for group work, and group climate. Educational Psychology, 25(4), 395-407. https://doi.org/10.1080/01443410500041706

Huff, L. C., Cooper, J. et Jones, W. (2002). The development and consequences of trust in student project groups. Journal of Marketing Education, 24(1), 24-34.

Izard, C. E., Dougherty, F. E., Bloxom, B. M. et Kotsch, N. E. (1974). The Differential Emotions Scale: a method of measuring the subjective experience of discrete emotions. [document inédit]. Nashville, Tenn. : Vanderbilt Univ.

Kapp, E. (2009). Improving student teamwork in a collaborative project-based course. College Teaching, 57(3), 139-143.

Miller, J. E., Trimbur, J. et Wilkes, J. M. (1994). Group dynamics: Understanding group success and failure in collaborative learning. New Directions for Teaching and Learning, $59,33-44$.

Moreno, J. L. (1965). Psychothérapie de groupe et psychodrame. Paris : Presses universitaires de France.

Oakley, B. A., Hanna, D. M., Kuzmyn, Z. et Felder, R. M. (2007). Best practices involving teamwork in the classroom: Results from a survey of 6435 engineering student respondents. IEEE Transactions on Education, 50(3), 266-272.

Oberlé, D. et Drozda-Senkowska, E. (2006). Processus orientés vers la tâche vs processus orientés vers le groupe : une vieille distinction toujours fructueuse ? Les Cahiers Internationaux de Psychologie Sociale, 70(2), 63-72. https://doi.org/10.3917/cips.070.0063

Ohland, M. W., Loughry, M. L., Woehr, D. J., Bullard, L. G., Felder, R. M., Finelli, C. J., Layton, R. A., Pomeranz, H. R. et Schmucker, D. G. (2012). The comprehensive assessment of team member effectiveness: Development of a behaviorally anchored rating scale for self and peer evaluation. Academy of Management Learning \& Education, 11, 609-630.

Raucent, B. et Vander Borght, C. (2006). Chapitre 3. Qu'est-ce qui a changé : quelles approches ont été mises en œuvre ?. Dans : Benoît Raucent éd., Être enseignant : Magister? Metteur en scène? (p. 165-236). Louvain-la-Neuve : De Boeck Supérieur. 
https://doi.org/10.3917/dbu.rauce.2006.01.0165"

Rosenberg, M. B. (1999). Les mots sont des fenêtres (ou des murs). Introduction à la Communication NonViolente. Genève : Éditions Jouvence.

Schaefer, A., Nils, F., Sanchez, X. et Philippot, P. (2010). Assessing the effectiveness of a large database of emotion-eliciting films: A new tool for emotion researchers. Cognition and Emotion 24(7), 1153-1172.

Verzat, C., O’Shea, N. et Raucent, B. (2015). Réguler le leadership dans les groupes d'étudiants en APP. Revue internationale de pédagogie de l'enseignement supérieur [En ligne], 31(1). URL : http:// journals.openedition.org/ripes/905 ; DOI :

https://doi.org/10.4000/ripes.905 
T

Modules "dynamique de groupe" du Projet en diagnostic environnemental.

\section{Consignes}

Cette évaluation est réalisée à la demande des assistants du cours LBIR1270 (Projet intégré en diagnostic environnemental). Elle concerne essentiellement la partie "Dynamique de groupe".

Les participations sont strictement anonymes et confidentielles.

Les résultats seront traités par Julie Lecoq, conseillère pédagogique au Louvain Learning Lab. Nous vous remercions pour votre collaboration.

Pour les questions suivantes, veuillez exprimer votre degré d'accord ou de désaccord sur une échelle à 4 niveaux (Pas du tout d'accord, Pas d'accord, D'accord, Tout à fait d'accord); SAUF si d'autres consignes sont indiquées.

\section{Perception du cours dans son ensemble (cours théorique, TP et modules sur la dynamique de} groupe)

2.1 De façon générale, j'ai apprécié ce cours et ses TP/ modules spécifiques

2.2 Les différentes parties du cours (cours théorique, TP et Modules sur la dynamique de groupe) s'articulent bien entre elles.

\section{Pas du tout d'accord}

Pas du tout d'accord
Tout à fait'accord

Tout à fait'accord

2.3 Concernant la complémentarité et la cohérence entre ces parties, je souhaiterais ajouter :

\section{Evaluation des Modules "Dynamique de groupe"}

\subsection{Globalement, j'ai apprécié les modules sur la dynamique} de groupe

Pas du tout d'accord
Tout à fait'accord

3.2 Les points que j'ai le plus appréciés dans ce module sont...

3.3 A mon avis, les points qui permettraient d'améliorer ce module sont ...

\section{Au sujet du fonctionnement de mon groupe :}

3.4 Je suis satisfait des membres de mon groupe

3.5 Je suis satisfait de la manière dont on a travaillé ensemble

\begin{tabular}{|c|c|c|c|c|c|}
\hline $\begin{array}{l}\text { Pas du tout } \\
\text { d'accord }\end{array}$ & $\square$ & $\square$ & $\square$ & $\square$ & $\begin{array}{l}\text { Tout à } \\
\text { fait'accord }\end{array}$ \\
\hline $\begin{array}{l}\text { Pas du tout } \\
\text { d'accord }\end{array}$ & $\square$ & $\square$ & $\square$ & $\square$ & $\begin{array}{l}\text { Tout à } \\
\text { fait'accord }\end{array}$ \\
\hline
\end{tabular}

$30 / 11 / 2020$, Page $1 / 4$ 


\section{Evaluation des Modules "Dynamique de groupe" [Continue]}

3.6 Je suis content d'avoir été dans ce groupe

3.7 Afin de compléter nos travaux, mes co-équipiers et moi devions compter les uns sur les autres pour des informations et des conseils

3.8 J'avais besoin de mes co-équipiers pour réaliser le travail

3.9 J'ai eu l'impression de travailler en solo

3.10 Mes co-équipiers et moi avons échangé et partagé des informations, des faits et des concepts

3.11 Mes co-équipiers et moi avons testé les idées du groupe

3.12 Mes co-équipiers et moi avons réfléchi ensemble pour restructurer nos connaissances et affiner notre compréhension

3.13 Mes co-équipiers et moi étions constamment focalisés sur l'objectif à atteindre

3.14 Mes co-équipiers et moi avons toujours respecté les délais imposés

3.15 Mes co-équipiers et moi avons produit un travail de qualité

3.16 Dans notre groupe, nous avons réussi à établir un climat de confiance

3.17 Dans notre groupe, nous avons géré de façon constructive les difficultés qui sont survenues
Pas du tout d'accord

Pas du tout d'accord

Pas du tout d'accord

Pas du tout d'accord

Pas du tout d'accord

Pas du tout d'accord

Pas du tout d'accord

Pas du tout d'accord

Pas du tout d'accord

Pas du tout d'accord

Pas du tout d'accord

Pas du tout d'accord
Tout à

fait'accord

Tout à

fait'accord

Tout à

fait'accord

Tout à

fait'accord

Tout à

fait'accord

Tout à

fait'accord

Tout à

fait'accord

Tout à

fait'accord

Tout à

fait'accord

Tout à

fait'accord

Tout à

fait'accord

Tout à

fait'accord

\section{Quelles sont les émotions caractérisant mon vécu en groupe ?}

Lors du travail de groupe, je me suis senti(e)...

3.18 attentif(ve), concentré(e), intéressé(e)

Jamais

$\square \square \square \square \square \square \square \quad$ Le plus souvent

3.19 en colère, irrité(e)

Jamais

Le plus

3.20 joyeux(se), amusé(e), gai(e)

Jamais

souvent

3.21 coupable, plein(e) de remords

Jamais

Le plus

souvent

3.22 apeuré(e), effrayé(e), craintif(ve)

Jamais

Le plus

souvent

3.23 gêné(e), embarrassé(e), honteux(se)

Jamais

Le plus

souvent

3.24 triste, cafardeux(se)

Jamais

Le plus

souvent

3.25 dégoûté(e), écoeuré(e)

Jamais

e plus

souvent

3.26 anxieux(se), tendu(e), nerveux(se)

Jamais

souvent

3.27 heureux(se), exalté(e), épanoui(e)

Jamais

Le plus

souvent

3.28 surpris(e), étonné(e)

Jamais

e plus

souvent

3.29 passionné(e), pleinement engagé(e)

Jamais

Le plus

souvent

ee plus

souvent 


\section{Mes compétences transversales}

Pour chaque objectif, estimez, selon vous, votre niveau de compétence $(0=$ pas du tout compétent; $10=$ très compétent), AVANT le cours et AU TERME du cours

Mener un projet en équipe, en planifiant les étapes et la coordination de ce projet en fonction d'objectifs prédéfinis et d'échéances qui sont imposées

\subsection{Avant le cours}

4.2 Après le cours

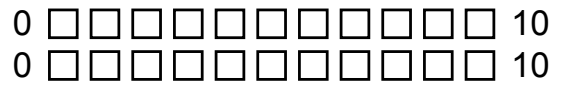

Communiquer par l'intermédiaire d'un rapport écrit les résultats et conclusions principaux relatifs à la réalisation d'un projet, en suivant un canevas rigoureux et en utilisant un style et une forme respectant les standards scientifiques

4.3 Avant le cours

$0 \square \square \square \square \square \square \square \square \square \square \square 10$

4.4 Après le cours

$0 \square \square \square \square \square \square \square \square \square \square \square 10$

Communiquer oralement le résultat d'un projet devant une audience
4.5 Avant le cours
$0 \square \square \square \square \square \square \square \square \square \square \square 10$
4.6 Après le cours
$0 \square \square \square \square \square \square \square \square \square \square \square 10$

Construire une dynamique relationnelle favorable dans une équipe de travail
4.7 Avant le cours
$0 \square \square \square \square \square \square \square \square \square \square \square 10$
4.8 Après le cours

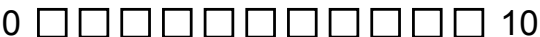

Mettre en place les conditions propices à une bonne dynamique d'équipe ultérieurement, dans d'autres travaux de groupe

\subsection{Avant le cours}

4.10 Après le cours

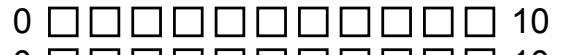

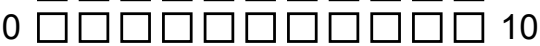

Me connaître et me donner les moyens d'évoluer personnellement

4.11 Avant le cours

$0 \square \square \square \square \square \square \square \square \square \square \square 10$

4.12 Après le cours

$0 \square \square \square \square \square \square \square \square \square \square \square 10$

Connaître les autres et ajuster mes interventions en tenant compte d'eux

\subsection{Avant le cours}

$0 \square \square \square \square \square \square \square \square \square \square \square 10$

4.14 Après le cours

$0 \square \square \square \square \square \square \square \square \square \square \square 10$

4.15 Autres éléments que je souhaite communiquer aux assistants qui ont encadré et animé les modules:

\section{Merci pour votre précieuse collaboration!}

